\title{
A review on the vibration analysis for a damage occurrence of a cantilever beam
}

\begin{abstract}
Identification of defects in structures and its components is a crucial aspect in decision making about their repair and total replacement. Failure to detect the faults has various consequences, and sometimes may lead to a catastrophic failure. The conducted research work reported analytical and experimental investigations on the effects of a crack on the cantilever steel beam with circular cross section. The objective of this review is to quantify and to determine the extent of the damage magnitude and the location of the cantilever beams. In analytical study, finite element method (FEA) software was used in developing the model. The results showed that, by monitoring the change of the natural frequency it is a feasible and viable tool to indicate the damage occurrence and magnitude. Unlike for small crack depth, the natural frequencies are not a good damage detector. Mode shapes indicated good sensitivity to detect the damage magnitude for all crack parameters. Frequency Reduction Index (FRI) and Modal Assurance Criteria (MAC) were found to be in order a feasible tool to find the magnitude of the damage in beam structures. While, Coordinate Modal Assurance Criteria (COMAC) and Curvature Change Index (CCI) were used to predict the location of the crack tested beams and proved to be feasible.
\end{abstract}

Keyword: Vibration analysis; Damage detection; Cantilever beam 